\title{
Superior dissecting capability of a new ultrasonic device improves efficiency and reduces adhesion formation
}

\author{
Alissa L Welling, Patrick J Scoggins, John F Cummings, Jeffrey W Clymer*, and Joseph F Amaral \\ Preclinical Research Center, Ethicon, Inc., Cincinnati OH, USA
}

\begin{abstract}
Background: Over the last two decades use of ultrasonic shears has grown in complex surgery where both precise dissection and meticulous hemostasis is required. With the introduction of HARMONIC ${ }^{\circledR} \mathrm{HD} 1000 \mathrm{i}$ Shears (HD1000i), surgeons can now depend upon a single instrument to perform minimally-invasive dissection while securely sealing vessels up to and including $7 \mathrm{~mm}$ in diameter. This study was performed to compare HD1000i to the previous version of the device (HAR7) in sealing, dissecting and adhesion formation.
\end{abstract}

Methods: Ex vivo testing in porcine tissues compared transection and marching speed, shaft temperature after repeated use, leaks at transection, seal reliability and burst pressures between HD1000i and HAR7. In an acute in vivo porcine study, initial hemostasis, hemostasis after a blood pressure challenge, thermal damage and dissecting ability were compared between HD1000i and HAR7. In a 30-day survival porcine study, initial hemostasis, seal durability with blood pressure challenge after the survival period, and adhesion formation were compared between the devices.

Results: HD1000i had significantly faster transection and marching speeds ( $<<0.001)$ than HAR7, and similar shaft temperature, leaks at transection, seal reliability and burst pressure. In acute and survival studies, HD1000i had a high rate of hemostatic seals both initially and after blood pressure challenge, and thermal damage was not significantly different from HAR7. HD1000i was rated as superior in dissecting capability and had a significantly lower rate of adhesion formation than the HAR7 ( $\mathrm{p}=0.005)$.

Conclusion: With an integrated hand piece, the new HD1000i shears can simplify operating room setup, and should lead to fewer instrument changes, as the device is designed to both to perform dissection and seal large vessels. The versatility of this ultrasonic device may lead to more efficient surgical procedures and improved patient outcomes.

\section{Introduction}

Fundamentally, surgeons look for three key attributes in all devices they use to perform any surgical procedure; efficiency, predictability/ reliability and minimal complications. Furthermore, as the complexity of surgical procedures increases, so too does the importance surgeon place on these attributes. This is perhaps most apparent in complex oncologic surgical procedures which tend to be long, laborious operations requiring meticulous and extensive lymph node dissections to improve staging and outcomes, relying on impeccable hemostasis to prevent the need for transfusion and obfuscation of the operative field, and subject to numerous postoperative complications related to the extensive dissection.

The requirement for maximum efficiency, precise hemostatic dissection for lymphadenectomy, and reliable hemostasis under all conditions including those of radiated tissue and post chemotherapy tissue, has made ultrasonic energy in general and Harmonic energy devices in particular, the preferred energy modality for complex oncologic surgery by many surgeons. The mechanism of action of ultrasonic energy allows not only for less lateral thermal damage but also for a smaller and more precise footprint due to the ability to simultaneously cut and coagulate tissue when compared to simpler sealing technologies such as advanced bipolar energy devices.

Despite the strong aspects noted above, ultrasonic devices also have drawbacks that must be considered, such as heat generation in the blade and reliability of vessel sealing with a traditional limit of $5 \mathrm{~mm}$ diameter vessel size for most devices. This has caused many surgeons to use a suite of instruments during a procedure, relying on Harmonic or ultrasonic devices for hemostatic dissection and yet another advanced bipolar device for sealing large vessels. Clearly such an approach is neither time nor cost efficient. Thus the past decade has seen significant improvements in Harmonic technology with the introduction of adaptive tissue technology that has resulted in heat management and advanced hemostasis. Given the ability of HARMONIC ACE ${ }^{\oplus}+7$ (HAR7) to seal up to $7 \mathrm{~mm}$ vessels with burst pressures equal to or greater than that of advanced bipolar devices, surgeons today can confidently choose one instrument for the entire procedure that will meet the needs for hemostatic dissection and complex hemostasis including vessels up to $7 \mathrm{~mm}$ in diameter [1].

It has also been known for quite some time that the efficiency of dissection and cutting would be enhanced if the jaws of the device were longer, more defined for dissection and the overall speed of transection and sealing were improved. Since their introduction in the early 1990's ultrasonic surgical cutting and coagulating devices have principally operated at a frequency of $55.5 \mathrm{kHz}$ [2]. Since the wavelength of

Correspondence to: Jeffrey W Clymer, Ethicon Inc., 4545 Creek Rd, Cincinnati OH 45242, USA, Tel: 513-337-3318; E-mail: jclymer@its.jnj.com

Key words: ultrasonic, dissection, harmonic, adhesions, vessel sealing

Received: January 04, 2017; Accepted: January 13, 2017; Published: January 16, 2017 
the device is inversely proportional to the frequency, only a change in frequency can substantially permit a change in wavelength and the corresponding working jaw length. Recently, a device has been developed, HARMONIC ${ }^{\circledR}$ HD 1000i Shears (HD1000i), that uses an operating frequency of $50 \mathrm{kHz}$, thereby allowing for a $30 \%$ increase in jaw length and a $37 \%$ increase in jaw opening while maintaining the ability to seal $7 \mathrm{~mm}$ vessels. The purpose of this study was to compare this new device, HD1000i, to the previous version, HAR7, to evaluate burst pressure and sealing reliability on various vessels sizes, transection time, instrument temperatures, hemostasis, lateral thermal damage and dissecting ability.

\section{Methods}

Devices used in this study were HARMONIC ${ }^{\circledR}$ HD 1000i Shears, either 20 or $36 \mathrm{~cm}$ length (HD1000i), and HARMONIC $\mathrm{ACE}^{\oplus}+7$ Shears, either 23 or $36 \mathrm{~cm}$ length (HAR7) powered by the GEN11 Generator (Ethicon, Inc., Cincinnati $\mathrm{OH})$.

The study device, HD1000i, has considerable differences in energy delivery compared to all previous Harmonic models in three respects. First is the aforementioned lower frequency of $50 \mathrm{kHz}$ compared to the $55.5 \mathrm{kHz}$ in all other Harmonic devices to date. Second, the piezoelectric ceramic discs are integrated into the device itself so there is no separate hand piece that must be attached (plug and play). Third, there are only two energy activation buttons rather than the three in the comparator HAR7 device; Advanced Hemostasis mode designed for vessels up to $7 \mathrm{~mm}$ in diameter and complex hemostasis, and the Energy Activation mode which merges the previous Minimum and Maximum modes into a single mode capable of dissecting avascular tissue and hemostatic transection of vessels up to and including $5 \mathrm{~mm}$. The HAR7 has been previously described in detail. ${ }^{1}$

\section{Benchtop testing}

Shaft temperature: The shaft temperature of HD1000i, $36 \mathrm{~cm}$ length, at power level 5, was compared to HAR7 after 20 transections per device in ex vivo porcine jejunum ( $\mathrm{n}=30$ per device). The devices were prepared by covering the distal 10 - $\mathrm{cm}$ of the shaft with matte black spray paint. The temperature-sensing infrared camera was mounted 10 $\mathrm{cm}$ from the device and focused on the distal $10 \mathrm{~cm}$ of the device.

Transection and marching speed: Transection time was determined by application of the devices to porcine carotid artery, 3-5 $\mathrm{mm}$ in outer diameter, with HD1000i using the Energy button at power level 5, or with HAR7 using the Min button at power level 3, and in vessels up to $7.0 \mathrm{~mm}$ in diameter with both HD1000i and HAR7 in Advanced Hemostasis mode ( $\mathrm{n}=10$ per device). Marching speed was assessed by determining the time necessary to transect $150 \mathrm{~mm}$ of porcine jejunum tissue using the Energy button at power level 5 for HD1000i and the Max button at power level 5 for HAR7 ( $n=30$ per device).

Burst pressure: Porcine carotid artery vessels of outer diameter 3-5 mm were sealed with HD1000i using the Energy button at power level 5, or with HAR7 using the Min button at power level 3 ( $n=60$ per device). Burst pressure was determined by infusing the sealed vessels with saline until leakage was observed. Each sealed and transected vessel yielded two sides for pressure testing; the lower of the burst pressures for each pair was used in the analysis. Seal reliability was determined by estimating the $95 \%$ lower confidence bound of seals with burst pressures of $240 \mathrm{~mm} \mathrm{Hg}$ or higher. Both sides of the seals were used for evaluation of seal reliability.

\section{In vivo evaluations}

All in vivo procedures were reviewed and animals approved for use in the study by the Institutional Animal Care and Use Committee.

\section{In vivo acute testing}

For acute evaluation, a total of 32 animals were used. Each of the HD1000i devices $(20$ and $36 \mathrm{~cm}$ ) and the HAR7 devices $(23$ and 36 $\mathrm{cm})$ were tested on 8 pigs each, with half of the animals used with the Advanced Hemostasis mode and half with the Energy Activation mode (or the Minimum/Maximum mode for the HAR7 devices). For the Advanced Hemostasis mode, 6 vessels per animal were used up to and including $7 \mathrm{~mm}$ in diameter, including gastroepiploic pedicle, short gastric pedicles, splenic artery and vein, and carotid artery. For the Energy Activation mode, 8 vessels per animal were used up to and including $5 \mathrm{~mm}$ in diameter, including gastroepiploic artery and veins, short gastric pedicles, splenic artery and vein and carotid artery. For the HAR7, Minimum mode was used for vessels of diameter 2 to 5 $\mathrm{mm}$, and Maximum mode for vessels less than $2 \mathrm{~mm}$ in diameter. All vessel seals were evaluated for hemostasis at initial application (firstpass hemostasis) and after a simulated hypertensive crisis, wherein the animal's blood pressure was increased to at least $200 \mathrm{~mm} \mathrm{Hg}$ for 10 minutes using phenylephrine as a vasopressor agent.

Surgical procedure: After a standard procedure of induction of anesthesia with Telazol/xylazine, and maintenance with isoflurane, with the pig in dorsal recumbency, an arterial catheter was placed to directly monitor blood pressure and a phenylephrine drip was administered if systolic blood pressure fell below $80 \mathrm{~mm} \mathrm{Hg}$. Splenectomy was performed through a midline laparotomy and carotid artery transection via a midline neck incision. During the splenectomy, the gastroepiploic pedicle or artery and veins, short gastric pedicles, and splenic artery and vein were isolated, the diameters were measured, and then the vessels were sealed and transected. The extent of thermal damage was assessed via histological evaluation of collagen denaturation of the carotid artery seal using hematoxylin and eosin staining.

To evaluate tissue dissection capability, the HD1000i was compared to HAR7 in non-energy, mechanical dissection of the iliac and lymph node in each animal. Each device was used to isolate the iliac artery from the iliac vein and surrounding tissue, and to mobilize a lymph node in the base of the small bowel mesentery. Assessment was based on a five-point comparison scale, in which the HAR1000i device was judged 1: notably superior, 2: slightly superior, 3: no different than, 4: slightly inferior, or 5: notably inferior to HAR7.

Separately, dissection was evaluated in thoracic applications. A thoracotomy was performed between the $4^{\text {th }}$ and $7^{\text {th }}$ intercostal space and the ribs were removed to expose the thoracic cavity. Following blunt dissection of the parietal surface, the test devices were used to skeletonize the pulmonary artery, to separate the azygous and pulmonary veins from the aorta, and to isolate the veins from the intercostal dorsal side branches. Whenever lymph nodes were encountered, the test devices were used to isolate them from the surrounding tissue.

\section{In vivo survival testing}

For survival evaluation, a total of 16 animals were used. The HD1000i and HAR7, both $36 \mathrm{~cm}$ in length, were tested on 8 pigs each, with half of the animals used with the Advanced Hemostasis mode and half with the Energy Activation mode (or the Minimum/Maximum mode for HAR7). For the Advanced Hemostasis mode, vessels were used up to and including $7 \mathrm{~mm}$ in diameter, including gastroepiploic 
pedicle, short gastric pedicles, splenic artery and vein, and carotid artery. For the Energy Activation mode, vessels were used up to and including $5 \mathrm{~mm}$ in diameter, including gastroepiploic artery and veins, short gastric pedicles, splenic artery and vein, and carotid artery. For the HAR7, Minimum mode was used for vessels of diameter 2 to $5 \mathrm{~mm}$, and Maximum mode for vessels less than $2 \mathrm{~mm}$ in diameter. All vessel seals were evaluated for hemostasis at initial application (first-pass hemostasis).

Surgical procedure: The approach and splenectomy were performed as in the acute evaluation. After splenectomy, either the right or left carotid artery was exposed, skeletonized and transected. After all transections and evaluations were completed, the incisions were closed using standard surgical technique with sutures and/or staples.

After the survival period of 30 days, and immediately prior to euthanasia, vessel seal integrity was challenged with the pig under general anesthesia using a vasopressor to increase blood pressure and to simulate an acute hypertensive crisis. Systolic blood pressure was raised to at least $200 \mathrm{~mm} \mathrm{Hg}$ for 10 minutes. After euthanasia, the integrity of all seals was individually confirmed during necropsy, and the presence of tissue adhesions surrounding the seals was observed and graded by a pathologist. Briefly the grading scale was; 0 : no adhesions, 1: localized thin adhesions, 2: localized thick adhesions, 3: multifocal thick adhesions, and 4: extensive thick adhesions.

\section{Statistical evaluation}

Comparisons of continuous variables were performed using Student's t-test, or in the case of non-normal distributions, via the Mann-Whitney test. Proportions were compared by Fisher's exact test. Attributes (i.e., dissection grading) were analyzed using the Wilcoxon signed rank test. For all tests, an alpha level of significance was taken as 0.05 .

\section{Results}

\section{Benchtop testing}

The HD1000i shaft temperature was not significantly different from that of HAR7 (Table 1). Using similar thickness tissue, the transection time for HD1000i was 35\% faster than for HAR7 for vessels of $5 \mathrm{~mm}$ or less, and $40 \%$ faster for vessels of $7 \mathrm{~mm}$ or less. Marching speed of transection through porcine jejunum was $37 \%$ faster with HD1000i. There were no significant differences between HAR7 and HD1000i in leaks at transection, burst pressure, and seal reliability up to $240 \mathrm{~mm}$ $\mathrm{Hg}$.

\section{In vivo acute evaluation}

There were no statistical differences between HD1000i and HAR7 in proportion of initial hemostasis or hemostasis at challenge in either the Energy Activation mode or Advanced Hemostasis mode (Table 2). The proportion of hemostatic seals was extremely high with only 1 failure of 239 seals for HD1000i and 1 failure of 224 for HAR7. There were also no differences in thermal damage between the devices for these groupings. In comparison to HAR7, the new HD1000i was rated as notably superior in iliac dissection and slightly superior in lymph node and thoracic dissection (Table 3).

\section{In vivo survival evaluation}

There were no statistical differences between HD1000i and HAR7 in proportion of initial hemostasis or hemostasis at challenge after the survival period in either the Energy Activation mode or Advanced Hemostasis mode (Table 4). There were no failures at the challenge following the survival period for either device. In comparison to HAR7, HD1000i had $42 \%$ fewer adhesion $(\mathrm{p}=0.005)$.

\section{Discussion}

The results of the present studies demonstrate that a small reduction in operating frequency from $55.5 \mathrm{kHz}$ to $50 \mathrm{kHz}$ can result in significant performance enhancements that should improve clinical efficiency, reliability and hemostasis. Specifically, the HD1000i outperformed HAR7 in many areas tested including perhaps most notably speed. HD1000i was $35 \%$ faster sealing vessels up to $5 \mathrm{~mm}$ in size and $40 \%$ faster in vessels up to $7 \mathrm{~mm}$, and marching time was $37 \%$ faster for HD1000i, with the same high burst pressures observed with HAR7. These changes however, are not solely due to frequency and the HD1000i has significant differences in maximum driving power

Table 1. Summary of Benchtop Comparisons of HD1000i and HAR7.

\begin{tabular}{|c|c|c|c|c|}
\hline Measure & HD1000i & HAR7 & Statistical Test & p-value \\
\hline Shaft Temperature (mean \pm stdev) & $66.3 \pm 2.0 \mathrm{C}$ & $64.7 \pm 9.2 \mathrm{C}$ & Student's t-test & 0.359 \\
\hline Transection Time Energy Mode (median) & $4.10 \mathrm{~s}$ & $6.27 \mathrm{~s}$ & Mann-Whitney & $<0.001$ \\
\hline Transection Time AdvHemo Mode (mean \pm stdev) & $9.19 \pm 0.83 \mathrm{~s}$ & $15.29 \pm 3.38 \mathrm{~s}$ & Student's t-test & $<0.001$ \\
\hline Marching Speed (median) & $3.81 \mathrm{~mm} / \mathrm{s}$ & $2.78 \mathrm{~mm} / \mathrm{s}$ & Mann-Whitney & $<0.001$ \\
\hline Leaks at Transection (proportion) & $2 / 120(1.7 \%)$ & $2 / 120(1.7 \%)$ & Fisher's Exact & $>0.999$ \\
\hline Burst Pressure (median) & $1448 \mathrm{~mm} \mathrm{Hg}$ & $1646 \mathrm{mmHg}$ & Mann-Whitney & 0.090 \\
\hline $95 \%$ Reliability at $240 \mathrm{~mm} \mathrm{Hg}$ & $98.20 \%$ & $98.40 \%$ & - & - \\
\hline Actual Reliability at $240 \mathrm{~mm} \mathrm{Hg}$ & $60 / 60(100 \%)$ & $60 / 60(100 \%)$ & Fisher's Exact & $>0.999$ \\
\hline
\end{tabular}

Table 2.Hemostasis and thermal damage in a porcine acute surgery model.

\begin{tabular}{|c|c|c|c|c|}
\hline Mode/Measure & HD1000i $(20 \& 36 \mathrm{~cm})$ & HAR7 $(23 \& 36 \mathrm{~cm})$ & Statistical Test & p-value \\
\hline \multicolumn{5}{|c|}{ Energy Activation Mode( $\leq 5 \mathrm{~mm}$ diameter $)$} \\
\hline Initial Hemostasis & $72 / 72(100 \%)$ & $64 / 64(100 \%)$ & Fisher's Exact & $>0.999$ \\
\hline Hemostasis at Challenge & $64 / 65(98 \%)$ & $64 / 64(100 \%)$ & Fisher's Exact & $>0.999$ \\
\hline Thermal Damage & $2.34 \pm 0.45 \mathrm{~mm}$ & $2.14 \pm 0.64 \mathrm{~mm}$ & Student's t-test & 0.318 \\
\hline \multicolumn{5}{|c|}{ Advanced Hemostasis Mode ( $\leq 7 \mathrm{~mm}$ diameter $)$} \\
\hline Initial Hemostasis & $54 / 54(100 \%)$ & $47 / 48(98 \%)$ & Fisher's Exact & $>0.999$ \\
\hline Hemostasis at Challenge & $48 / 48(100 \%)$ & $48 / 48(100 \%)$ & Fisher's Exact & $>0.999$ \\
\hline Thermal Damage & $2.06 \pm 0.45 \mathrm{~mm}$ & $2.05 \pm 0.40 \mathrm{~mm}$ & Student's t-test & 0.984 \\
\hline
\end{tabular}


Table 3.Comparisons between HD1000i and HAR7 devices in iliac, lymph node and thoracic dissection.

\begin{tabular}{|l|c|c|c|}
\hline $\begin{array}{l}\text { Comparison, } \\
\text { HD1000i (20/36 cm) vs HAR7 } \\
\text { (23/36 cm) }\end{array}$ & $\begin{array}{c}\text { Iliac } \\
\text { Dissection }\end{array}$ & $\begin{array}{c}\text { Lymph Node } \\
\text { Dissection }\end{array}$ & $\begin{array}{c}\text { Thoracic } \\
\text { Dissection }\end{array}$ \\
\hline HD1000i notably superior (1) & 29 & 0 & 4 \\
\hline HD1000i slightly superior (2) & 3 & 32 & 9 \\
\hline No difference (3) & 0 & 0 & 3 \\
\hline HD1000i slightly inferior (4) & 0 & 0 & 0 \\
\hline HD1000i notably inferior (5) & 0 & 0 & 0 \\
\hline Median & 1.0 & 2.0 & 2.0 \\
\hline Wilcoxon signed rank test & $\mathrm{p}<0.001$ & $\mathrm{p}<0.001$ & $\mathrm{p}=0.001$ \\
\hline $\mathrm{H}_{0}: \tilde{\mathrm{x}}=3.0$ & & & \\
\hline
\end{tabular}

Table 4.Hemostasis and adhesions in a porcine survival model.

\begin{tabular}{|c|c|c|c|c|}
\hline Mode/Measure & $\begin{array}{l}\text { HD1000i (36 } \\
\mathrm{cm})\end{array}$ & $\begin{array}{c}\text { HAR7 (36 } \\
\text { cm) }\end{array}$ & $\begin{array}{c}\text { Statistical } \\
\text { Test }\end{array}$ & p-value \\
\hline \multicolumn{5}{|c|}{ Energy Activation Mode( $\leq 5 \mathrm{~mm}$ diameter $)$} \\
\hline Initial Hemostasis & $32 / 32(100 \%)$ & $32 / 32(100 \%)$ & Fisher's Exact & $>0.999$ \\
\hline Hemostasis at Challenge & $32 / 32(100 \%)$ & $32 / 32(100 \%)$ & Fisher's Exact & $>0.999$ \\
\hline \multicolumn{5}{|c|}{ Advanced Hemostasis ( $\leq 7 \mathrm{~mm}$ diameter $)$} \\
\hline Initial Hemostasis & $22 / 24(92 \%)$ & $23 / 24(96 \%)$ & Fisher's Exact & $>0.999$ \\
\hline Hemostasis at Challenge & $24 / 24(100 \%)$ & $24 / 24(100 \%)$ & Fisher's Exact & $>0.999$ \\
\hline \multicolumn{5}{|l|}{$\begin{array}{l}\text { Adhesions (Both } \\
\text { Groups) }\end{array}$} \\
\hline $\begin{array}{l}\text { 0: None } \\
\text { 1: Thin } \\
\text { 2: Thick } \\
\text { 3: Multifocal } \\
\text { 4: Extensive }\end{array}$ & $\begin{array}{c}22 \\
17 \\
1 \\
0 \\
0\end{array}$ & $\begin{array}{c}9 \\
24 \\
7 \\
0 \\
0\end{array}$ & & \\
\hline Proportion of Adhesions & $18 / 40(45 \%)$ & $31 / 40(78 \%)$ & Fisher's Exact & 0.005 \\
\hline
\end{tabular}

as necessitated by a longer blade and a very different blade geometry. Given a 30\% increase in jaw length and therefore tissue bite length, this speed increase should be expected.

Our studies also demonstrate HD1000i has superior dissecting ability compared to HAR7 in iliac, lymph node and thoracic dissections. It is important to recognize that a weakness of this finding is its subjective nature and the small sample size. Nevertheless, the result is consistent with the redesign of the instrument tips that allow for greater mechanical leverage due to a $30 \%$ greater jaw length and $37 \%$ greater jaw opening, the greater curvature allowed by longer length and the precise taper of the tip for entrance between tissue planes (Figure 1).

A critically important finding of these studies is that there were no differences noted in histological lateral thermal damage in tissues divided by HD1000i or HAR7 despite significantly greater power and a broader, longer blade base for HD1000i compared to HAR7. Ultrasonic devices have long been valued for the little lateral thermal damage they produce and recognized for significant clinical benefits that potentially accrue from this de minimis tissue damage. For example, a recent metaanalysis on the use of Harmonic devices for dissection in mastectomy for breast cancer in comparison to electrosurgery showed Harmonic provided less postoperative drainage, seroma development, blood loss and wound complications [3]. Harmonic was regarded as superior to electrosurgery for axillary dissections, demonstrating shorter duration of drainage, lower rates of seroma formation and reduced numbness $[4,5]$. Likewise in pancreatic surgery, Harmonic dissection is associated with lower rates of pancreatic fistula and abdominal abscess [6,7]. In dissection during D2 lymphadenectomy following gastrectomy, Harmonic technology reduced operative time, blood loss and drainage volume [8]. Finally, Harmonic is particularly valuable in dissection near
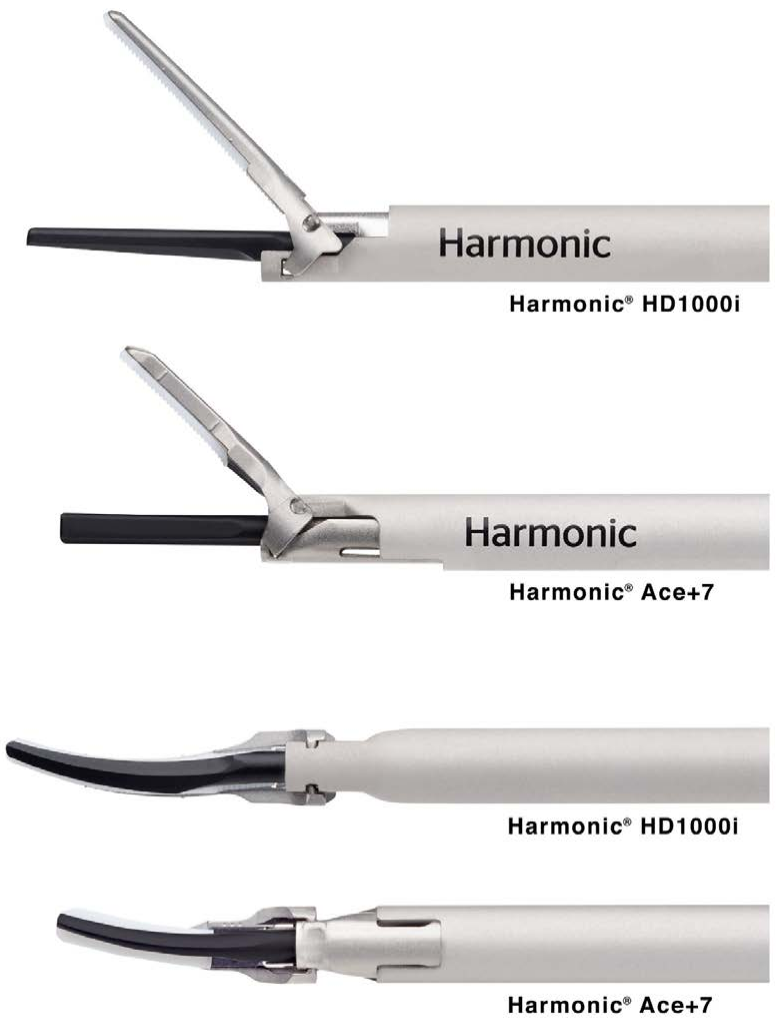

Figure 1. The Harmonic ${ }^{\circledR}$ HD 1000 i and Harmonic ${ }^{\circledR}$ Ace +7 Shears in the open position demonstrating the wider and longer jaw of HD1000i (top) and a view of the underside of both instruments (bottom).

nerves, where electrosurgery has been shown to cause nerve damage, yet the use of Harmonic is no more injurious than cold scalpel $[9,10]$.

Perhaps a remarkable finding of these studies is not only the reduction in adhesions observed in this study for HD1000i compared to HAR7, but even more so the absolute low frequency of adhesions in either group. An association however between the use of ultrasonic energy and adhesion formation has been reported in preclinical studies since shortly after the introduction of Harmonic. The first study to report this finding evaluated the use of Harmonic technology in a laparoscopic cholecystectomy model comparing ultrasonic energy with electrosurgery and laser [11]. In that study, the ultrasonic device demonstrated a two-thirds reduction in adhesion formation compared to electrosurgery [12]. Models of adhesion found reasons for an expectation of fewer adhesions, with a very significant difference in pathological adhesion scores favoring a previous version of the ultrasonic scalpel [13]. In a rat model, microscopic adhesion scores for an ultrasonic device were similar to the control, whereas macroscopic adhesion scores for ultrasonic were significantly lower than those for a bipolar device and for suture ligation [14]. More recent evaluations found fewer adhesions for a new ultrasonic device with intelligent control of energy delivery [15]. Such findings may in part be related to the lower thermal damage created with ultrasonic energy and a reflection of a similar mechanism associated with seroma, drainage and pain noted previously. On the other hand, these preclinical studies demonstrating a reduction in adhesion formation should not be considered as strong support for reduction in human adhesion formation in the absence of clinical data to support such a finding. 
In conclusion, these data support the belief that HD1000i should provide enhanced clinical efficiency in complex surgical producers where longer tissue bites and enhanced dissecting ability are at a premium with hemostatic reliability at least equivalent to the best in class products available today. These attributes bring closer the reality of a multifunctional device that can be used for each step of a complex procedure without compromise. While this preclinical work is promising, these findings will need to be validated in clinical studies.

\section{References}

1. Timm RW, Asher RM, Tellio KR, Welling AL, Clymer JW, et al. (2014) Sealing vessels up to $7 \mathrm{~mm}$ in diameter solely with ultrasonic technology. Medical devices 7: 263-271.

2. Amaral JF (1994) The experimental development of an ultrasonically activated scalpel for laparoscopic use. Surg Laparosc Endosc 4: 92-99. [Crossref]

3. Huang J, Yu Y, Wei C, Qin Q, Mo Q, et al. (2015) Harmonic Scalpel versus Electrocautery Dissection in Modified Radical Mastectomy for Breast Cancer: A MetaAnalysis. PloS one 10: e0142271. [Crossref]

4. Malik S, Zarkoon N, Ishaqui H, Dar U (2015) Axillary dissection in mastectomy: Ultracision vs monopolar electrocautery. Pak J Med Health Sci 9: 1328-1330.

5. Nawaz A, Waqar S, Khan A, Mansoor R, Butt UI, et al. (2015) Harmonic Scalpel Versus Electrocautery in Axillary Dissection in Carcinoma Breast. JCPSP 25: 870-873.

6. Uzunoglu FG, Stehr A, Fink JA, Izbicki JR (2012) Ultrasonic dissection versus conventional dissection techniques in pancreatic surgery: a randomized multicentre study. Annals of surgery 256: 675-680.

7. Lei H, Xu D, Shi X, Han K (2016) Ultrasonic Dissection versus Conventional
Dissection for Pancreatic Surgery: A Meta-Analysis. Gastroenterology research and practice 2016.

8. Cheng H, Hsiao CW, Clymer JW, Schwiers ML, Tibensky BN, et al. (2015) Gastrectomy and D2 Lymphadenectomy for Gastric Cancer: A Meta-Analysis Comparing the Harmonic Scalpel to Conventional Techniques. Int J Surg Oncol 2015: 397260. [Crossref]

9. Chen C, Kallakuri S, Vedpathak A, Chimakurthy C, Cavanaugh JM, et al. (2012) The effects of ultrasonic and electrosurgery devices on nerve physiology. Br J Neurosurg 26: 856-863. [Crossref]

10. Chen C, Kallakuri S, Cavanaugh JM, Broughton D, Clymer JW (2015) Acute and subacute effects of the ultrasonic blade and electrosurgery on nerve physiology. British J Neurosurg 29: 569-573.

11. Amaral JF (1994) Ultrasonic dissection. Endosc Surg Allied Technol 2: 181-185. [Crossref]

12. Amaral J, Chrostek C (1997) Experimental comparison of the ultrasonically-activated scalpel to electrosurgery and laser surgery for laparoscopic use. Minim Invasive Ther Allied Technol 6: 324-331.

13. Vetere PF, Lazarou G, Mondesir C, Wei K, Khullar P, et al. (2011) Strategies to minimize adhesion formation after surgery. JSLS 15: 350-354.

14. Kucuk GO, Ertem M, Kepil N (2013) Histopathological Response and Adhesion Formation After Omentectomy with Ultrasonic Energy, Bipolar Sealing, and Suture Ligation. Ind J Surg 77: 799-804.

15. Broughton D, Welling AL, Monroe EH, Pirozzi K, Schulte JB, et al. (2013) Tissue effects in vessel sealing and transection from an ultrasonic device with more intelligent control of energy delivery. Medical devices 6: 151-154.

Copyright: $\odot 2017$ Welling AL. This is an open-access article distributed under the terms of the Creative Commons Attribution License, which permits unrestricted use, distribution, and reproduction in any medium, provided the original author and source are credited. 